\author{
PREPRINT OF REVISED PAPER \\ to be published in Thin Solid Films \\ Special Issue from Second International Conference on Spectroscopic \\ Ellipsometry
}

\title{
High-Speed, High-Accuracy Optical Measurements of Polycrystalline Silicon for Process Control
}

Tyrone E. Benson, Arun Ramamoorthy, Leonard I. Kamlet, and Fred L. Terry, Jr.

Department of Electrical Engineering \& Computer Science, 111 DTM Bldg., The University of Michigan, 2360 Bonisteel Blvd., Ann Arbor, MI 48109-2108

\author{
Corresponding Author: \\ Fred L. Terry, Jr. \\ Department of EECS \\ University of Michigan \\ 118C DTM Building \\ 2360 Bonisteel Blvd.
}

Ann Arbor, Mi. 48109-2108

phone: $313-763-9764$

fax: 313-936-0347

e-mail: fredty@umich.edu 


\section{High-Speed, High-Accuracy Optical Measurements of Polycrystalline Silicon for Process Control}

Tyrone E. Benson, Arun Ramamoorthy, Leonard I. Kamlet, and Fred L. Terry, Jr.

Department of Electrical Engineering \& Computer Science, 111 DTM Bldg., The University of Michigan, 2360 Bonisteel Blvd., Ann Arbor, MI 48109-2108

Keywords: surface roughness, polycrystalline silicon, etch rate monitoring, process control

\section{Abstract}

Highly accurate non-contact polycrystalline silicon (poly-Si) film thickness measurements are important for both real-time feedback control and run-to-run control. Both spectroscopic ellipsometry (SE) and normal-incidence spectral reflectometry (SR) are complicated by poly-Si surface effects and variable bulk poly-Si refractive indices. In this paper we will describe an empirical modification of Beckmann/Kirchhoff scattering theory to account for the effects of rough layers in SR. We will present results from in situ monitoring of reactive ion etching of poly$\mathrm{Si} / \mathrm{SiO}_{2} / \mathrm{Si}$, and ex situ comparisons to TEM and AFM. Preliminary conclusions on the applications of this model to SE analysis of poly-Si will also be presented.

\section{Introduction}

Highly accurate non-contact polycrystalline silicon (poly-Si) film thickness measurements are important for both real-time feedback control and run-to-run control. Both spectroscopic ellipsometry (SE) and normal-incidence spectral reflectometry (SR) are complicated by poly-Si surface roughness effects ${ }^{1,2,3,4}$ and variable bulk poly-Si refractive indices. ${ }^{5,6}$ The accuracy of poly-Si thickness measurements is usually more strongly limited by the models employed than raw measurement noise or instrument calibration errors. These model-related systematic errors directly limit the minimum time required for extraction of in situ etch rates because the thickness change over this minimum time must be larger than the systematic and random errors. ${ }^{7}$ We will present a 
simple scalar scattering theory method for modeling the poly-Si surface roughness effects and will present results using this model in high-speed poly-Si reactive ion etch (RIE) rate monitoring. We will present quantitative comparisons between in situ SR data and ex situ TEM and AFM measurements that show significant improvements in the accuracy of both bulk film and surface roughness thicknesses obtained using this model vs. the commonly employed Bruggemann effective media approximation (BEMA) model for surface roughness. Improvements in the dynamic response of the etch rate monitor will be shown. We will also discuss the use of the $\mathrm{B} / \mathrm{K}$ model for SE measurements of poly-Si and improvements in the models for the bulk poly-Si dielectric function.

\section{Experimental Details}

We use a low-cost Ocean-Optics ${ }^{\mathrm{TM}} \mathrm{CCD}$ spectrometer/W-halogen lamp system to perform high-speed, in situ normal incidence SR measurements over the 400-800nm wavelength range on poly-Si/ $\mathrm{SiO}_{2} / \mathrm{Si}$ samples during reactive ion etching . The details of this system, accuracy comparisons to a commercially available spectrophotometer, thickness accuracy tests vs. SE on $\mathrm{SiO}_{2} / \mathrm{Si}$ samples, and its initial applications to high-speed in situ monitoring of poly-Si RIE have been previously presented. ${ }^{7,8} \mathrm{SR}$ data with $5 \mathrm{~nm}$ resolution can be collected in $40 \mathrm{~ms}$ with good raw signal-to-noise ratios. Ex situ spectroscopic ellipsometry data was collected using a Sopra GESP-5 variable-angle rotating polarizer ellipsometer run in the tracking analyzer mode.

Analysis of both SR and SE data was done using the Levenberg-Marquardt approach. We will report the quality of fits using the unbiased estimator, $\zeta$, given by:

$$
\zeta=\sqrt{\sum_{\lambda_{i}}\left(R_{\text {measured }}-R_{\text {model }}\right)^{2} /(N-P)}
$$

for reflectometry and by:

$$
\zeta=\sqrt{\sum_{\lambda_{i}}\left\{\left(\tan (\psi)_{\text {measured }}-\tan (\psi)_{\text {model }}\right)^{2}+\left(\cos (\Delta)_{\text {measured }}-\cos (\Delta)_{\text {model }}\right)^{2}\right\} /(2 N-P)}
$$


for ellipsometry. In the above $\mathrm{R}$ is the reflectance (intensity ratio), $\mathrm{N}$ is the total number of wavelength points, $\mathrm{P}$ is the number of model parameters in the regression, and the summation is done over the measured wavelength points, $\lambda_{\mathrm{i}}$. For comparison purposes, analysis of a $\sim 600 \mathrm{~nm}$ $\mathrm{SiO}_{2}$ on $\mathrm{Si}$ sample film yielded an oxide thickness of $607.5 \pm 0.43 \mathrm{~nm}\left(\zeta=5.60 \times 10^{-2}\right)$ using the GESP-5 and a thickness of $604.13 \pm 4.19 \mathrm{~nm}\left(\zeta=1.47 \times 10^{-2}\right)$ using our SR system when the same SE-derived $\mathrm{SiO}_{2}$ reference file was used for both cases. Analysis of a $\sim 8 \mathrm{~nm} \mathrm{SiO} / 2 \mathrm{Si}$ sample using the GESP-5 over the $270-800 \mathrm{~nm}$ range yielded $\zeta=6.19 \times 10^{-3}$. For analysis of SR data over the 400 to $800 \mathrm{~nm}$ range, we modeled the refractive index vs. wavelength of our poly-Si using the BEMA assuming a binary mixture amorphous $\mathrm{Si}(\mathrm{a}-\mathrm{Si})$ and crystalline $\mathrm{Si}(\mathrm{c}-\mathrm{Si})$ with a screening factor $(q=0.5)$ appropriate to the columnar grain structure of $625^{\circ} \mathrm{C}$ poly-Si $i^{9}$ or using data extracted from smooth samples analyzed by SE.

Poly-Si/SiO $/ 2$ Si samples were prepared in our laboratory using $100 \mathrm{~mm} \mathrm{Si} \mathrm{wafers} \mathrm{and}$ conventional microelectronic processing techniques. $\mathrm{SiO}_{2}$ films were grown by thermal oxidation in $\mathrm{O}_{2}$ at $1000^{\circ} \mathrm{C}$. Poly-Si was deposited by $\mathrm{SiH}_{4}$-based low pressure chemical vapor deposition (LPCVD) at $625^{\circ} \mathrm{C}$ and a typical pressure of 200 mTorr. Reactive ion etch experiments were performed in a $13.56 \mathrm{MHz}$ Applied Materials 8300 Hexode reactor in a $\mathrm{CF}_{4} / 5 \%$ Ar or $\mathrm{CF}_{4} / 5 \%$ $\mathrm{Ar} / \mathrm{O}_{2}$ ambient at a pressure of $20 \mathrm{mT}$ Torr and an RF power of $1000 \mathrm{~W}$.

Cross-sectional TEM (XTEM) photographs were taken on a JEOL 2000FX electron microscope with a $200 \mathrm{KV}$ acceleration voltage. samples were prepared for XTEM by mechanical grinding and polishing followed by ion milling to final thickness. Atomic force microscopy (AFM) was done on a Digital Instruments NanoScope III in tapping mode using a silicon tip cantilever.

\section{Results and Discussion}

Following the work of Carniglia ${ }^{10}$, we use a modified Beckmann/Kirchhoff diffraction $(\mathrm{B} / \mathrm{K})$ theory model with 2 empirical modifying factors to account for reflectance losses which result from non-specular scattering of light by the rough surfaces of poly-Si films: 


$$
r_{\text {total }}=f_{1}\left[r_{01} \exp \left(-2 k^{2} n_{0}^{2} \cos ^{2}\left(\phi_{0}\right) \sigma^{2}\right)+f_{2} \frac{t_{01} t_{10} \exp \left[-2 k^{2}\left(N_{0} \cos \left(\phi_{0}\right)-N_{1} \cos \left(\phi_{1}\right)\right)^{2} \sigma^{2}\right] r_{12} e^{-j 2 \beta}}{1-r_{01} \exp \left(-2 k^{2} N_{1}^{2} \cos ^{2}\left(\phi_{1}\right) \sigma^{2}\right) r_{12} e^{-j 2 \beta}}\right]
$$

In this expression, $f_{1}$ and $f_{2}$ are empirical constants, $\mathrm{k}=2 / \lambda, \sigma$ is the RMS surface roughness thickness, $\mathrm{N}_{0}$ and $\mathrm{N}_{1}$ are the complex refractive indices of the ambient and the top layer respectively, and the remaining Fresnel coefficients and other terms have the usual meanings. ${ }^{11} \mathrm{~A}$ similar formulation has been used to study diamond film growth. ${ }^{12}$ This formula is written in the form for an ambient/rough film/substrate system, but can be easily generalized to a system of multilayer smooth films with a rough top film by replacing $r_{12}$ with the complex reflection coefficient for the smooth layer film stack. For normal incidence applications, the cosine terms are both 1, and the Fresnel coefficients are polarization independent; for off-normal incidence applications (such as ellipsometry), the Fresnel coefficients for s or p polarization are evaluated as usual. With $f_{1}=f_{2}=1$, the usual $\mathrm{B} / \mathrm{K}$ scattering formulation results. In our application of this model, we treat these empirical factors as adjustable parameters in the same way as the more physically significant $\sigma$. We assume that these empirical factors are independent of polarization; therefore, only $f_{2}$ has an effect on ellipsometry measurements, as $f_{1}$ has the same effect on the total $\mathrm{s}$ and $\mathrm{p}$ reflectances.

Use of this formulation is supported by examining the reflection vs. wavelength of a thick poly-Si sample as shown in Fig. 1. Due to absorption in the bulk film, the region between 400nm and roughly $540 \mathrm{~nm}$ is dominated by the primary reflection from the top surface with very little thin film interference effects. Plotting $\ln \left(\mathrm{R} / \mathrm{R}_{0}\right)$ vs. $\mathrm{k}^{2}$ in this range yields a straight line as indicated in Fig. 2. The slope of the best fit line yields an RMS roughness of $32.3 \mathrm{~nm}$. The smooth film reflectance, $\mathrm{R}_{0}$, was calculated from a BEMA fit to thinner films of our poly-Si. We observed a consistent trend of a non-zero intercept of this best fit line (the unmodified theory would yield a zero intercept). This is accounted for by the empirical factor $f_{l}$ in our model, and could be due to errors in our calculated value of $\mathrm{R}_{0}$ vs. $\lambda$ or to calibration errors in our reflection measurement. 
Errors in the assumed $\mathrm{R}_{0}$ may result due to differences in the surface layer and bulk dielectric functions as well as the presence of thin native oxides. The factor $f_{2}$ is more difficult to link to physical factors. We included this factor to due to an ad hoc observation that it improves the fit of peak/valley amplitudes in our poly-Si measurements. It may be related to errors in the bulk poly-Si dielectric function, a different the dielectric function for the surface roughness layer vs. the bulk poly-Si, or the shape of the surface features.

A regression fit of the entire data range of Fig. 1 was done using an optical model of a B/K-rough poly-Si (BEMA)/48.5nm $\mathrm{SiO}_{2} / \mathrm{Si}$, and yielded the fit which also shown in Fig. 1. The quality of the fit is very good, as indicated by an unbiased estimator of $\zeta=5.15 \times 10^{-3}$. An attempt to fit the same data using a BEMA model for the surface roughness layer yielded a visually inferior fit (particularly in the oscillatory region), a very high surface roughness layer thickness (66.6nm), and a $\zeta=1.43 \times 10^{-2}$. This fit could be improved by including a scaling factor (equivalent to $f_{1}$ ) for reflectometer calibration error, but the resulting best fit occurred with the scaling factor at an unreasonably small value of 0.88 (12\% measurement error). Tests of our reflectometry system and procedures on $\mathrm{SiO}_{2} / \mathrm{Si}$ samples have shown that scaling errors of over $\pm 5 \%$ are physically unreasonable. For the $\mathrm{B} / \mathrm{K}$ model, the surface roughness for the overall fit agrees well with the shorter wavelength fit in Fig. 2; however, the extracted a-Si fraction of $2.1 \%$ for the poly-Si bulk is very low compared to that obtained from measurements of thinner samples. If the empirical factors $f_{1}$ and $f_{2}$ are held constant at 1 , a visually similar fit can be obtained with $\zeta=6.63 \times 10^{-3}$ and very nearly the same RMS surface roughness and bulk poly-Si thickness, but with an a-Si fraction of essentially $0\left(1.5 \times 10^{-5} \pm 1.06 \times 10^{-3}\right)$. This indicates a strong correlation between the empirical factor $f_{2}$ and errors in the bulk poly-Si dielectric function. It is also possible that part of this error is due to a difference between the dielectric response of the top surface vs. the bulk poly-Si. We will discuss this possibility in more detail later in this paper. For our more typical application of monitoring the RIE etching of poly-Si, we use films of $\sim 550 \mathrm{~nm}$ or less, and obtain better matches 
between the SR- and SE-derived a-Si fractions. ${ }^{8}$ For these films we obtain bulk a-Si fractions of $\sim 7 \%$ with SR and $\sim 9 \%$ with SE.

For thicker poly-Si films ( 500nm), good surface roughness data could be obtained using the BEMA approximation for the bulk poly-Si. For thinner films, the lack of an interference free region caused too much parameter correlation induced error. To study the validity of the $\mathrm{B} / \mathrm{K}$ separately from the suspect bulk BEMA model, we performed SE measurements at multiple angles of incidence $\left(76^{\circ}-70^{\circ}\right.$, each $\left.1^{\circ}\right)$ on a poly-Si film deposited under similar conditions to our films that had been smoothed by chemical-mechanical polishing (CMP). We fit the SE data using a 4oscillator complex harmonic oscillator approximation (CHOA) for the poly-Si. ${ }^{13}$ After obtaining the poly-Si thickness and approximate dielectric response, we then performed a direct extraction of the dielectric constants on a point by point basis.

We then made preliminary investigations using XTEM and AFM to verify the bulk film thickness and surface roughness accuracy. Five samples were investigated. All began as $\sim 660 \mathrm{~nm}$ poly-Si/SiO $/ 2 \mathrm{Si}$, and were etched for varying times in the AMAT 8300 . Four of the etched samples were examined by XTEM, and four were characterized for RMS surface roughness by AFM. The remaining film thicknesses and surface roughnesses were extracted from static SR measurements. The poly-Si dielectric constants obtained from SE as described above were used in both the B/K model and BEMA fits. BEMA estimates were based on a multilayer model consisting of a (BEMA of voids \& bulk poly-Si q=0.5)/poly-Si/SiO$/ 2 / \mathrm{Si}$. The results are shown in Table 1 . The agreement for both XTEM film thickness and AFM surface roughness with the B/K model SR is good in all cases. The BEMA results are generally inferior and show more random behavior in the surface roughness layer.

For monitoring of the remaining poly-Si film thickness and the etch rate during RIE, we desire as simple a model as possible to permit high-speed data analysis. In order to obtain etch rate data that reflects very rapid changes, it is necessary to have a data analysis technique which produces very small relative errors between sampled points. For high speed monitoring of rates, this can easily translate into a requirement of relative errors requirements of $<0.2 \mathrm{~nm}$. The effects of 
surface roughness can produce systematic errors of $>1 \mathrm{~nm}$ in the bulk film thickness. ${ }^{14}$ These then lead to oscillations or apparent noise when the film thickness vs. time data is numerically differentiated to obtain etch rates, as can been seen in our earlier work. ${ }^{7, \text { Error! Bookmark not defined.8,14 }}$

We have employed the $\mathrm{B} / \mathrm{K}$ model to reduce this problem. Our normal procedure is to model the poly-Si BEMA of a-Si and c-Si and to fit the bulk poly-Si thickness, a-Si fraction, and the $\mathrm{B} / \mathrm{K}$ parameters $\sigma, f_{1}$, and $f_{2}$ on data collected before the etch has begun. We then fix the a-Si fraction and $f_{2}$ as constants for the sample. When possible, we replace the BEMA bulk model with more accurate dielectric function data. For the data collected during etching, we fit the bulk film thickness and RMS surface roughness, $\sigma$, at each time step. The parameter $f_{1}$ is sometimes allowed vary to account for minor changes in the SR instrumentation calibration and minor window changes during the run, but its value generally stays at $1.00 \pm 0.05$ or better. Better fits to the SR data at each individual time step can, of course, be obtained by fitting all the model parameters in an attempt to account for changes in the sample surface texture which we have previously shown to occur during etching ${ }^{8}$. However, we have found that numerical correlation between the model parameters will lead to non-physical oscillations in the surface roughness terms which again lead to false oscillations in the derived etch rates. We had previously observed the same effect when using the BEMA model for surface roughness. An example of the use of this technique is shown in Fig. 3. A very smooth resulting thickness vs. time curve results. Numerical differentiation with a 31-point (15 s time window), 2nd order Savitsky-Golay algorithm yield a reasonably smooth etch rate curve. The sharp change in the etch rate at approximately 500s was the result of an intentional introduction of $\mathrm{O}_{2}$ into the $\mathrm{CF}_{4} / \mathrm{Ar}$ ambient and is easily resolved. Some possibly non-physical oscillations in the etch rate occur when the poly-Si film thickness nears 0 , possibly due to small roughness errors.

Our initial attempts to use the same optical model for SE analysis were unsuccessful with poor quality fits (particularly in the $\cos (\Delta)$ curves). There are several possibilities which may explain this problem including: (1) failure of the $\mathrm{B} / \mathrm{K}$ algorithm at strongly off-normal angles of 
incidence; (2) effects of depolarization in the reflected beam due to the surface roughness; and, (3) a difference between the dielectric response of the surface roughness layer and the bulk poly-Si. Data supporting the last possibility has been presented previously. ${ }^{3,4}$ We then examined a film similar to the previously discussed CMP-smoothed one in its as-deposited condition with SE at $70,72.5$, and $75^{\circ}$. Use of the $\mathrm{B} / \mathrm{K}$ model with the directly-obtained dielectric response did not yield good fits. We then applied a two layer model to the poly-Si , a rough top film on bulk poly-Si. The $\mathrm{B} / \mathrm{K}$ model with $f_{1}=f_{2}=1$ was then used to account for the surface roughness effects, and a 4oscillator CHOA for the top surface dielectric constant. The bulk poly-Si was modeled using the directly extracted dielectric function from the CMP-smoothed sample. The CHOA parameters for the bulk poly-Si were used as the initial guesses for the parameters for the dielectric function of the surface roughness layer. Regression yielded an good fit $\left(\zeta=2.31 \times 10^{-2}\right)$ to the as-deposited poly-Si as is shown in Fig. 4 with a top layer thickness of $14.7 \pm 0.5 \mathrm{~nm}$ with $\sigma=10.6 \pm 1.0 \mathrm{~nm}$, a bulk polySi thickness of $558.6 \pm 0.4 \mathrm{~nm}$, on a $7.97 \mathrm{~nm} \mathrm{SiO}$ film on $\mathrm{Si}$ (from an $\mathrm{SE}$ measurement of a monitor wafer). The extracted dielectric response of the bulk poly-Si and surface layer are shown in Fig. 5. The fit, while subject to significant possible sources of error, shows a structure at least qualitatively consistent with what would be expected for a poly-Si film with lower crystallinity and lower density. Also, use of a more conventional BEMA model (voids \& a-Si \& c-Si) for the surface roughness layer (with or without the $\mathrm{B} / \mathrm{K}$ scattering model) yields a very similar dielectric function for the surface layer (virtually identical peak values of $\varepsilon_{1}$ and $\varepsilon_{2}$ but with the peaks somewhat blue-shifted vs. the CHOA/BK model function).

Given the relatively large number of adjustable parameters (16 oscillator parameters, $\sigma$, and the bulk poly-Si thickness), a high quality fit is to be expected. However, we have used the top surface and bulk dielectric constants to improve the fits to our SR data on as-deposited films. For example, by applying the multilayer model, (top layer with $\mathrm{B} / \mathrm{K}$ roughness)/(bulk poly- $\mathrm{Si}) / \mathrm{SiO}{ }_{2} / \mathrm{Si}$ (with the top layer and bulk poly-Si dielectric functions from the SE analysis described above), to 
the data from sample 1 of table 1, we obtain the following fits: (i) $\sigma=14.1 \pm 0.8 \mathrm{~nm}$, top layer

thickness of $14.0 \pm 0.1 \mathrm{~nm}$, bulk poly-Si thickness of $638.6 \pm 0.3 \mathrm{~nm}, \zeta=2.60 \times 10^{-2}$ with $f_{1}$ and $f_{2}$ held constant at 1 ; and, (ii) $\sigma=17.8 \pm 0.1 \mathrm{~nm}$, top layer thickness of $19.2 \pm 0.2 \mathrm{~nm}$, bulk poly-Si

thickness of $634.8 \pm 0.1 \mathrm{~nm}, f_{1}=1.065 \pm 0.001, f_{2}=0.941 \pm 0.008, \zeta=2.45 \times 10^{-2}$. We have also used this model and dielectric function data sets to obtain good fits to poly-Si samples produced under similar conditions in our laboratory and two separate industrial laboratories. The modified dielectric response of the surface layer might also explain the a-Si fraction errors observed in thick poly-Si samples and possibly some of the residual oscillation in our etch rate monitoring.

\section{Conclusions}

Use of a relatively simple Beckmann/Kirchhoff scattering formulation with two empirical modifying factors can yield superior results for normal-incidence reflection surface roughness measurements of poly-Si vs. the commonly employed BEMA method. When this method is used with accurate dielectric function data for the bulk poly-Si film, SR data can yield very accurate surface roughness and total film thickness. It is directly useful in normal incidence reflection monitoring of poly-Si etching. Our preliminary analysis suggests that the empirical factors which we have introduced serve to make the extractions of both bulk poly-Si and RMS surface roughness thicknesses from reflection data more robust to small errors in the bulk poly-Si dielectric function and possibly to the presence of a top layer with a modified dielectric function. It is possible that these empirical factors would be unnecessary if the dielectric functions of the poly-Si and top layer were known to adequate accuracy. However, this level of sample analysis may not be practical for process control applications, particularly for RIE processes during which the nature of near surface region may be changing with time due to chemical and ion bombardment effects. The $\mathrm{B} / \mathrm{K}$ method shows some promise for use in SE analysis, but additional work is needed to verify and quantify the near-surface dielectric function behavior of the as-deposited poly-Si. 


\section{Acknowledgments}

We would like to acknowledge the support of the Semiconductor Research Corporation for the support of this work under contract no. 96-MC-085, the State of Michigan Center for Display Technology for use of the Sopra GESP-5 and other laboratory facilities. We would also like to

thank Dr. Joseph Bendik and National Semiconductor Corp. for some of the poly-Si samples used in this work. 


\section{References}

${ }^{1}$ I. Ohlidal, F. Lukes, Optica Acta, 19 (1972) 817.

${ }^{2}$ I. Ohlidal, F. Lukes, K. Navratil, Surf. Sci., 45 (1974) 91.

${ }^{3}$ G. E. Jellison, Jr., M. Keefer, L. Thornquist, Mat. Res. Soc. Symp. Proc., 283 (1993) 561.

${ }^{4}$ G. E. Jellison, M. F. Chisholm, S. M. Gorbatkin, Appl. Phys. Lett., 62 (1993) 3348.

${ }^{5}$ W. A. McGahan, B. R. Spady, B. D. Johs, and O. Lappara, Proc. SPIE, 2725 (1996) 450.

${ }^{6}$ A. Borghesi, M. E. Giardini, M. Marazzi, A. Sassella, G. De Sanit, Appl. Phys. Lett., 70 (1997) 892.

${ }^{7}$ T. E. Benson, L. I. Kamlet, S. M. Ruegsegger, C. K. Hanish, B. A. Rashap, P. Klimecky, J. S. Freudenberg, J. W. Grizzle, P. P. Khargonekar, and F. L. Terry, Jr., J. Vac. Sci. Technol. B, 14 (1996) 483.

${ }^{8}$ T. E. Benson, L. I. Kamlet, P. Klimecky, and F. L. Terry, Jr., J. Electron. Mat., 25 (1996) 955.

${ }^{9}$ S. Logothetidis, J. Appl. Phys., 65 (1989) 2416.

${ }^{10}$ C. K. Carniglia, Opt. Eng., 18 (1979) 104.

${ }^{11}$ see for example, R.M.A. Azzam and N.M. Bashara, Ellipsometry and Polarized Light, NorthHolland (1987) p. 286

${ }^{12}$ C. D. Zuiker, D. M. Gruen, A. R. Krauss, J. Appl. Phys., 79 (1996) 3541.

${ }^{13}$ F. L. Terry, Jr., J. Appl. Phys., 70, (1991) 409.

${ }^{14}$ T. E. Benson, Ph.D. Disseratation, University of Michigan (1996). 


\section{Figure Captions}

1. Reflectance vs. wavelength data for $\sim 1.6 \mu \mathrm{m}$ Poly-Si/48.5nm $\mathrm{SiO}_{2} / \mathrm{Si}$ sample (points) and a fit (solid curve) using the $\mathrm{B} / \mathrm{K}$ theory discussed in this paper. The fit yielded $\zeta=5.15 \times 10^{-3}$ with the fitted parameters: poly-Si thickness $1618.4 \pm 0.06 \mathrm{~nm}$ with an a-Si fraction of $0.021 \pm 0.0004$; $\sigma=31.44 \pm 0.02 \mathrm{~nm} ; \mathrm{f}_{1}=0.996 \pm 0.0004 ;$ and $\mathrm{f}_{2}=1.231 \pm 0.005$. A visually similar fit can be obtained with $\mathrm{f}_{1}=\mathrm{f}_{2}=1$, yielding $\zeta=6.63 \times 10^{-3}$ with the fitted parameters: poly-Si thickness $1621.1 \pm 0.18 \mathrm{~nm}$ with an a-Si fraction of $1.5 \times 10^{-5} \pm 1.06 \times 10^{-3} ; \sigma=31.47 \pm 0.23 \mathrm{~nm}$.

2. The shorter wavelength data of Figure $1(\lambda<537 \mathrm{~nm})$ plotted as $\ln \left(R / R_{0}\right)$ vs. wavenumber $k$. The measured data is plotted as individual points and the solid curve is a best fit straight line.

3. Remaining poly Si film thickness and etch rate vs. RIE etch time. The pre-etched sample consisted of ( $570 \mathrm{~nm}$ poly-Si)/(32.1 $\left.\mathrm{nm} \mathrm{SiO}_{2}\right) / \mathrm{Si}$. A directly-extracted poly-Si dielectric function was used in this analysis. SR data were sampled every $0.5 \mathrm{~s}$. A $0.5 \mathrm{sccm} \mathrm{O}_{2}$ flow was added to the $30 \mathrm{sccm} \mathrm{CF}_{4} / 5 \%$ Ar flow at $\sim 500$ s.

4. Measured (solid curves) and modeled (dashed curves) SE data at $75^{\circ}$ incidence for an asdepositied poly Si film using a combination of the B/K scattering model and a CHOA for the surface dielectric response.

5. The extracted dielectric response of the CMP-smoothed poly Si (solid curves) and the B/KCHOA-extracted as deposited surface layer (dashed curves). 


\section{Table Caption}

1. Comparison of XTEM and AFM measurements with SR data for as-deposited and RIE etched poly films. 


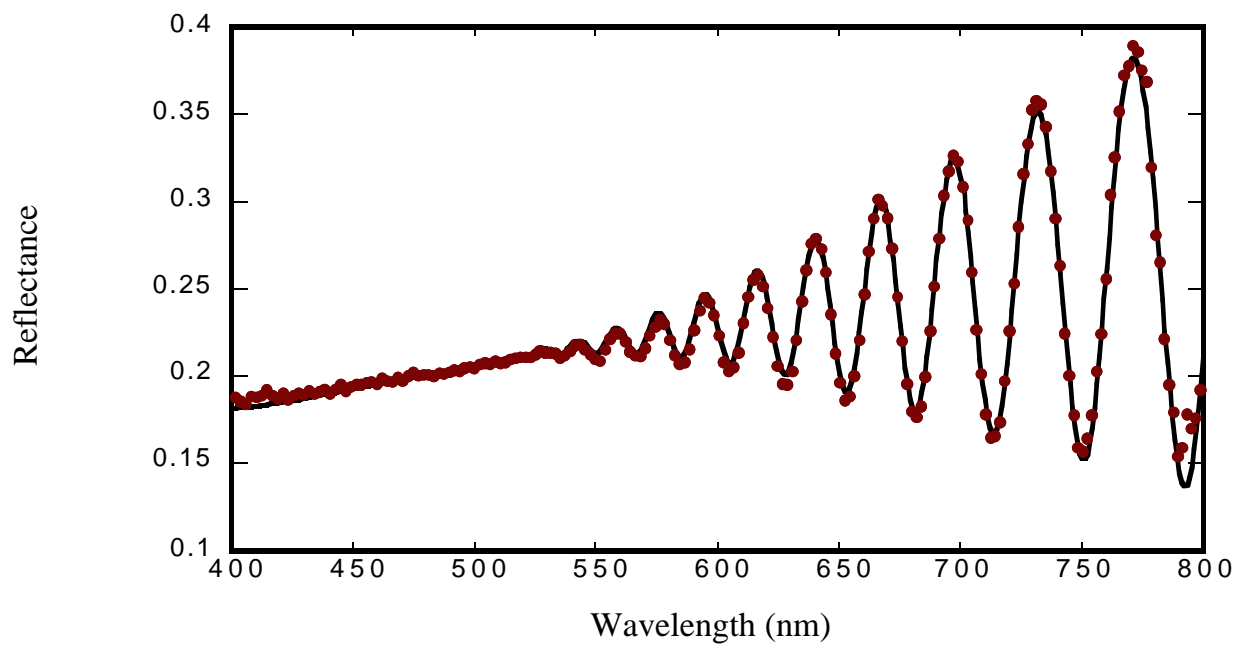

Fig. 1.

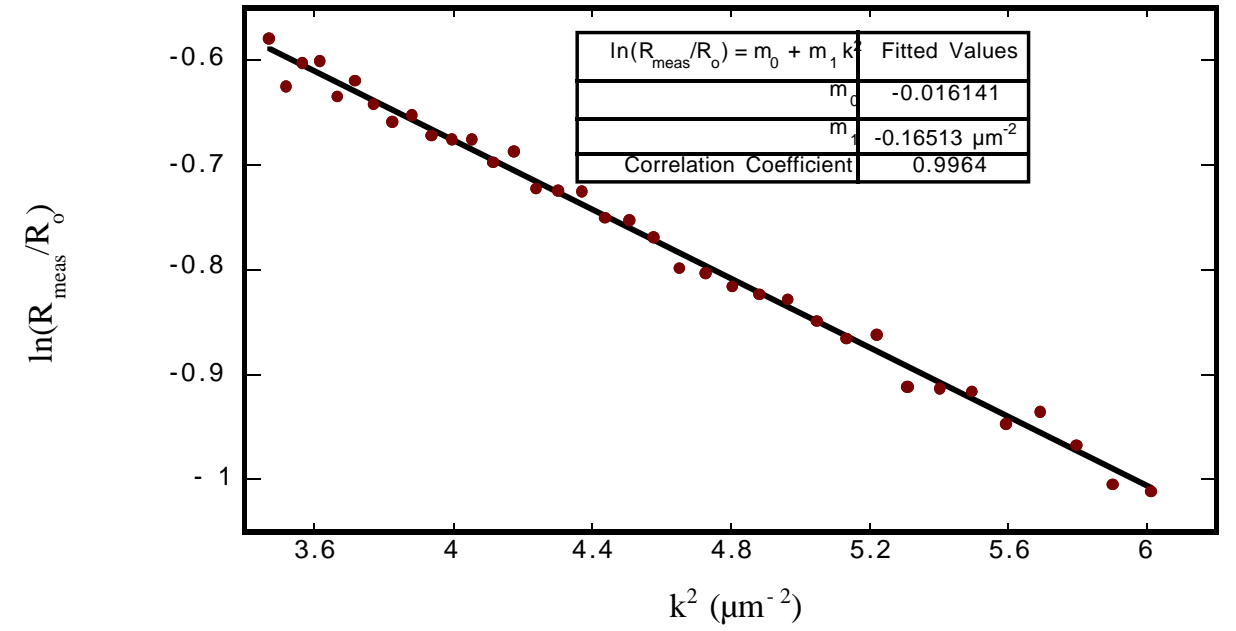

Fig. 2. 


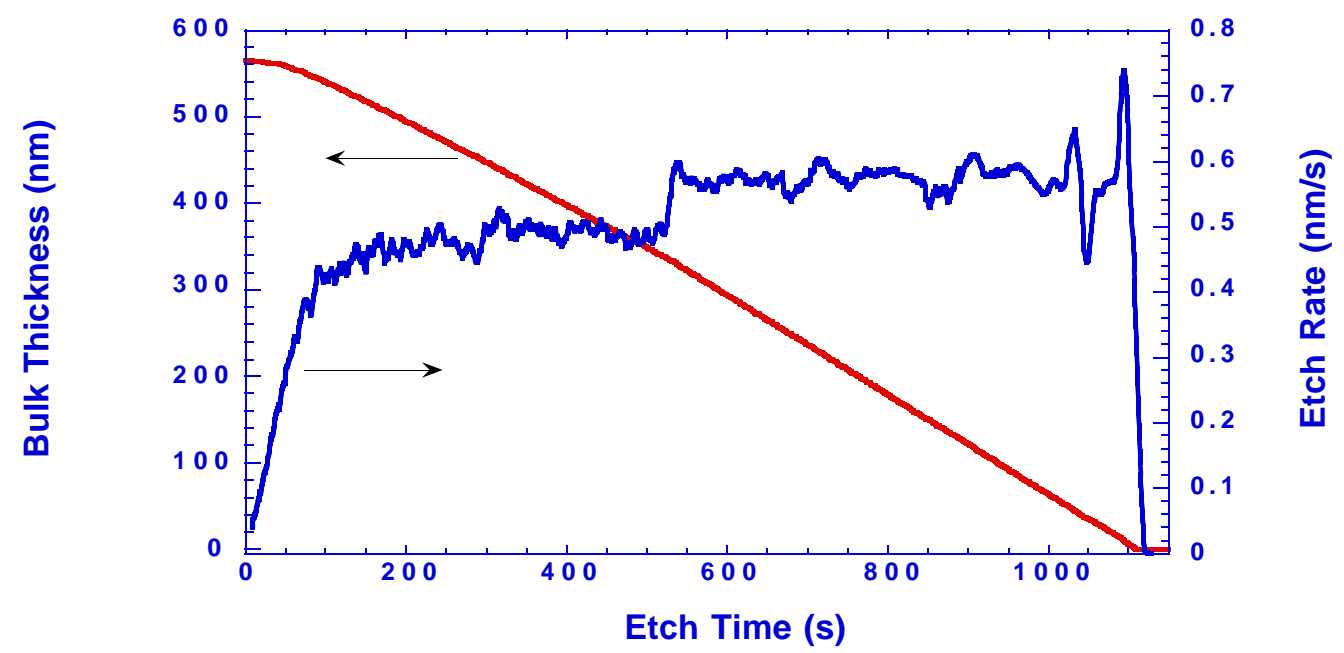

Fig. 3.

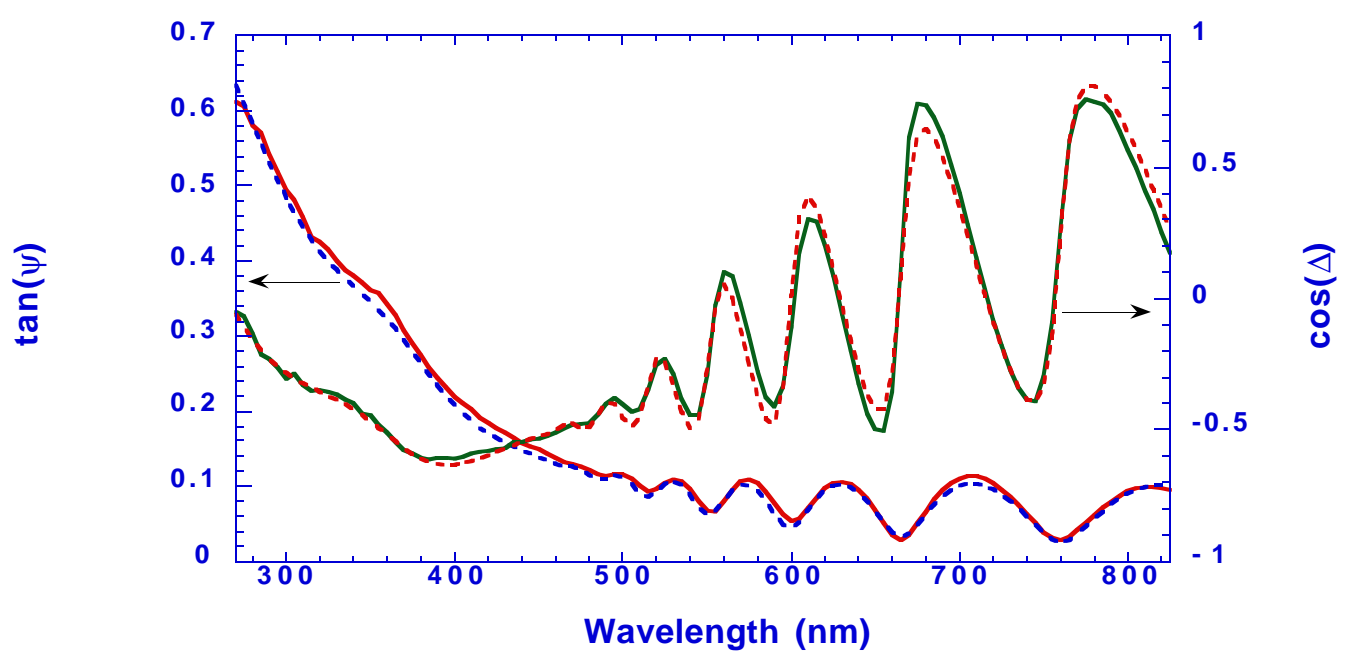

Fig. 4. 


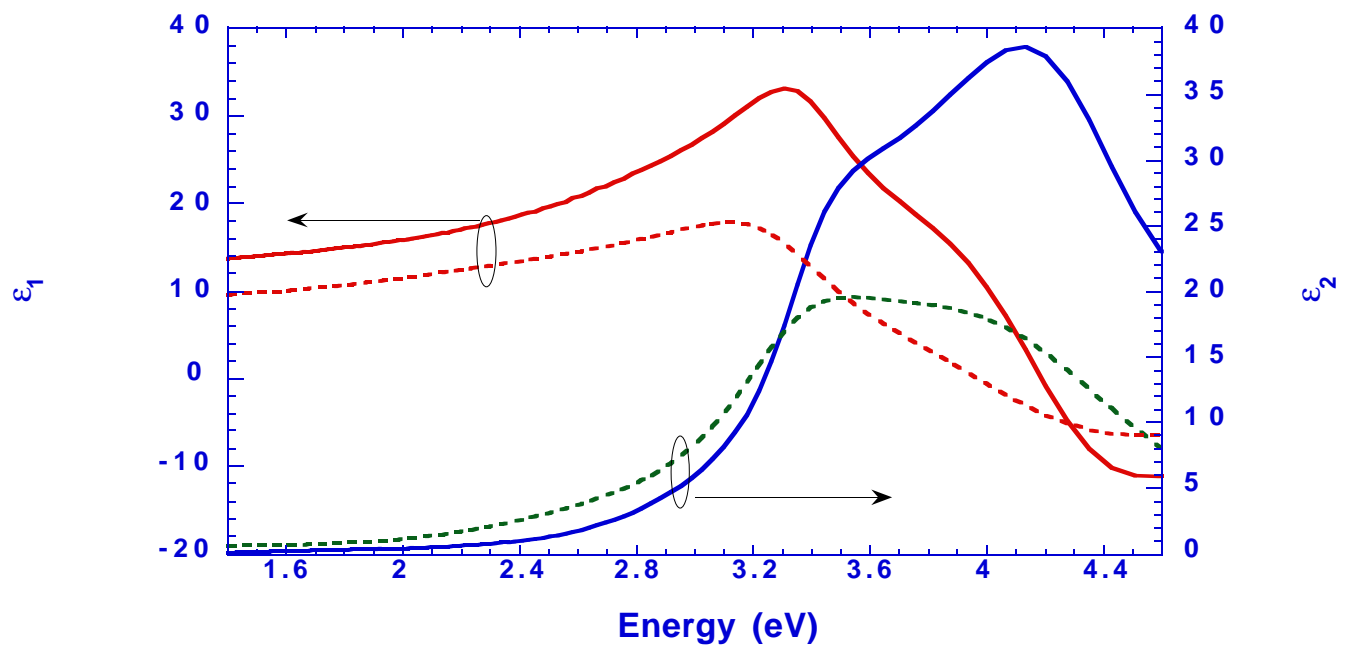

Fig. 5.

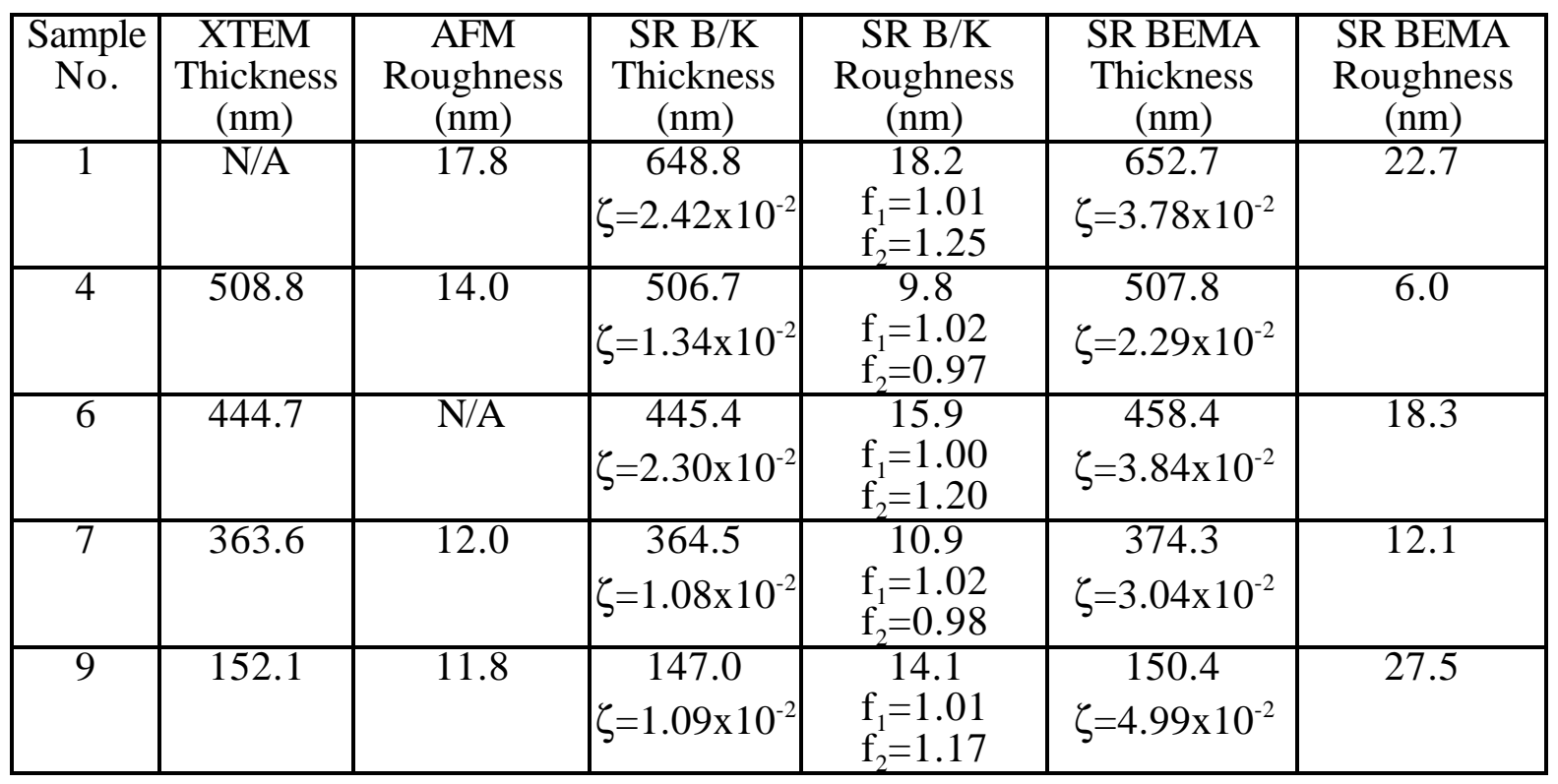

Table 1. 